\title{
7 Rituales
}

La selección y jerarquización de literatura se realiza, no obstante, mediante procedimientos concretos como la evaluación escrita que realizan los lectores, la inclusión en antologías como la de Granta, los encuentros, congresos y mesas redondas, la asignación de premios, la creación y participación en polémicas, las reseñas celebratorias, las entrevistas, etc. Bien puede ser, aunque no siempre sea así, que todos estos mecanismos resulten articulados, aunque sea de modo indirecto, desde y en función de un espacio de gestión centralizado como el que representa la casa matriz de un grupo multimedios. Como hemos visto, la producción de literatura mundial, al requerir una eficiencia mayor, procura sacar el mayor provecho posible por medio de una administración centralizada y racionalizada de estos recursos.

Por su parte, en su estudio sobre la función de los gatekeepers, William Marling recurre al concepto interaction rituals, de Randall Collins, para analizar procesos mediante los cuales escritores como Haruki Murakami, Paul Auster y Gabriel García Márquez llegaron a ingresar en el corpus de la literatura mundial. Los rituales de interacción, en tanto actos rituales de fundación de alianzas estratégicas, permiten explicar cómo se generan objetos simbólicos a partir de determinadas pertenencias sociales o redes de solidaridad. Mediante estos actos rituales, los participantes también van a adquirir el capital social y simbólico con el que se va a ir labrando una trayectoria en el campo. Al margen de las subclasificaciones que va a establecer luego, Collins propone que

The following are the ingredients of any interaction ritual:

1. a group of at least two people is physically assembled;

2. they focus attention on the same object or action, and each becomes aware that the other is maintaining this focus;

3. they share a common mood or emotion. (22)

Agreguemos, además, que, puesto que el concepto proviene de la microsociología, las situaciones concretas, la escenificación y la performance, van a ser el principal objeto de análisis de esta teoría. La propuesta de Collins es, al respecto, concluyente: "Let us begin at the site of all action: the local situation. All events take place in a here-and-now as concrete and particular", anota en su libro sobre las redes de interacción en el campo de la filosofía (20).

Los premios literarios, con el habitual acto de entrega, pueden ser, por lo tanto, entendidos como rituales de interacción ejemplares. Desde mediados del siglo XX la cantidad e importancia de los premios concedidos por editoriales españolas ha ido en aumento. El Premio Nadal, otorgado por la editorial Destino

Ә Open Access. (c) 2019 Jorge J. Locane, published by De Gruyter. (c))BY-NC-ND This work is licensed under the Creative Commons Attribution-NonCommercial-NoDerivatives 4.0 License. 
(ahora como parte de Planeta) desde 1944, vale por fundacional. Mientras que el Premio Planeta, que se entrega desde 1952, es, con 600000 euros, hasta el momento el más dotado del mundo, solo superado por el Premio Nobel. Como he comentado en el apartado anterior, los premios que poseen mayor impacto en la circulación de literatura latinoamericana a nivel mundial son los que ofrecen estas editoriales, particularmente Planeta y sus subsidiarias, como Seix Barral y el Premio Biblioteca Breve, Penguin Random House Mondadori y el Premio de Alfaguara, y el Premio Herralde de Anagrama. Voy a dedicarle mayor atención a este último en el próximo capítulo, me interesa destacar en este momento que, en tanto rituales de interacción, estos premios constituyen potentes herramientas para la producción de literatura mundial y para la asignación de prestigio, es decir, para su jerarquización. Acentuemos, no obstante, que en vista de la literatura que acá interesa, esta asignación de prestigio posee un carácter restrictivo y diferido. Mientras que el Premio Nobel distingue tanto narradores, como poetas y cronistas o ensayistas, los premios comerciales españoles de importancia se limitan al género novela con lo que al mismo tiempo establecen un recorte en la efectiva producción y condicionan la práctica de escritura. Establecen un recorte en la medida en que, de la diversidad que pueda haber en potencial circulación, la novela va a resultar claramente privilegiada y, en consecuencia, va a ser la que reciba por lejos mayor atención internacional. Condicionan la escritura en tanto que los escritores que pretendan conseguir cierta trascendencia internacional van a tener que cumplir con el "requisito" de ofrecer al público, en algún momento de su carrera, alguna novela. Esto se debe a que el tipo de prestigio que conceden estos premios supone implícitamente que un escritor, para que pueda ser reconocido como tal y para que pueda tener pretensiones de mundialidad, produce fundamentalmente novelas.

El segundo aspecto a considerar de estos decisivos rituales de interacción consiste en lo que James English (282) ha denominado desterritorialization of prestige. Este fenómeno se funda en una creciente dificultad de las localidades periféricas para asignar prestigio a sus productos simbólicos. Con la consolidación de la actual fase de la globalización, las localidades han cedido capacidad para valorar sus objetos estéticos y culturales, mientras que actores de carácter global se autoatribuyen una función valorativa, desplazada espacialmente, que responde a su propio programa de política cultural. De este modo, las grandes editoriales con sede central en España, de manera ejemplar mediante el otorgamiento de premios, han ganado importancia en la asignación de valor a productos literarios que van a entrar en circulación internacional como "latinoamericanos". En concreto, las ceremonias de entrega de premios operan como vitrinas donde las editoriales españolas -las que cuentan con recursos suficientes- hacen un llamado público para revelar al mundo los "grandes 
descubrimientos" de la literatura latinoamericana que ellas mismas, mediante los actores concretos que las representan, han seleccionado de acuerdo con sus criterios valorativos y su propia agenda de intereses. En lo que sigue, me interesa examinar con mayor detalle un acto ritual concreto, pero algo diferente, de elaboración de literatura mundial.

Anota Marling que

One obvious tactic to gain attention is to pick a quarrel with someone else, and we know that this often works in literature. A dispute will usually gain the attention of the one attacked and it might attract a crowd. But if everyone does this, no crowd gathers. Some arguments have greater appeal because they contradict the positions held by several people. If there are others who feel similarly, they gather round and provide support. (7)

Una polémica, precisamente, es lo que se intentó promover en junio de 2003 en Sevilla. Impulsado por el editor general de Seix Barral, Adolfo García Ortega, en la sede de la fundación José Manuel Lara, el evento titulado Primer Encuentro de Autores Latinoamericanos convocó, por una parte, a trece escritores de origen latinoamericano y, por la otra, a periodistas culturales españoles. De los trece escritores, dos participaron en calidad de "invitados especiales", mientras que los otros once conformaron el grupo núcleo en torno al cual giró el encuentro. Esta separación responde, por lo pronto, a razones generacionales: los once participantes principales, nacidos en los años 60 y 70, representaban a "los escritores jóvenes de América”, mientras que los dos restantes pertenecían a generaciones anteriores. Los escritores convocados fueron los siguientes: Guillermo Cabrera Infante, Roberto Bolaño, Jorge Franco, Rodrigo Fresán, Santiago Gamboa, Gonzalo Garcés, Fernando Iwasaki, Mario Mendoza, Ignacio Padilla, Edmundo Paz-Soldán, Cristina Rivera Garza, Iván Thays y Jorge Volpi. Todos, en calidad de voceros de la literatura latinoamericana, tuvieron su espacio para exponer en torno al estado y a las tendencias del momento en la literatura latinoamericana. Las ponencias, a su vez, fueron recogidas en el libro publicado por el sello Seix Barral en enero de 2004 Palabra de América.

Considerado como un ritual de interacción prototípico con el fin inmediato de introducir en el mercado internacional un relevamiento generacional, el encuentro presenta muchos aspectos de interés para pensar los mecanismos de producción de literatura latinoamericana mundial. Como en todo ritual, aquí también los roles están claramente establecidos: Cabrera Infante va a oficiar de autoridad o gurú, de figura ampliamente legitimada por la tradición, que ofrece su aprobación a los nuevos aspirantes. Bolaño, por su parte, actúa como figura de engarce entre ambas generaciones y como refuerzo simbólico que, con su propia experiencia, avala y autoriza una reconceptualización de los modelos de escritura "latinoamericana". Los once escritores restantes, en aquel momento 
todavía en proceso de ingreso o consolidación en el mercado internacional, van a presentar argumentos, siempre alineados bajo pautas de renovación ya preestablecidas por Bolaño, para ser admitidos como parte de la comunidad de escritores latinoamericanos reconocida a nivel internacional.

Algunos elementos merecen ser destacados. El grupo Planeta, encarnado para el caso por la fundación Lara y la editorial Seix Barral -la gran promotora y capitalizadora del boom en tiempos de Carlos Barral-, va a ser el proveedor de los recursos económicos necesarios para la escenificación. Muchos de los escritores convocados, como el mismo Cabrera Infante, como Volpi, Garcés y Mendoza con sus respectivos premios Biblioteca Breve (1999, 2000 y 2002), ya estaban vinculados a la editorial y al grupo previamente. Los otros, al menos a una de las grandes editoriales con sede en España. Este dato no se le pasa a Cabrera Infante cuando en el prólogo a Palabra de América, "Cita en Sevilla", comenta publicaciones de los escritores convocados y señala: "(Hay que decir que las diversas editoriales son todas españolas)” (14). El lugar concreto de realización del ritual posee también su significado e importancia: la fundación Lara en Sevilla. A pesar de que, en principio, se trata de un evento cultural que compromete ante todo a la escritura latinoamericana, el evento se lleva a cabo en un espacio que responde a un actor privado, con sus naturales intereses comerciales, y en una ciudad desplazada geográficamente del lugar del enunciado hacia la metrópoli como lugar de enunciación. De este modo, la "palabra de América" que reproduce el volumen se va a pronunciar bajo un régimen de producción del orden de lo privado y desde una locación geográfica que restituye enlaces y determinaciones coloniales. Si, además, se tiene en cuenta que la mayoría de los escritores del evento no tenían su lugar de residencia en América Latina y que estaban representados por agentes de Barcelona, ${ }^{1}$ se debe admitir que la "literatura latinoamericana" de la que pretendían dar cuenta el encuentro y el posterior volumen es en gran medida una construcción metropolitana, propia del nivel de la literatura mundial y no necesariamente en consonancia con las evoluciones de las literaturas locales.

\footnotetext{
1 Prácticamente todos los escritores que participaron del encuentro tenían a su agente en Barcelona. Obsérvese que las variables generación y género introducen matices interesantes en el patrón. La lista es la siguiente: Guillermo Cabrera Infante (Carmen Balcells), Roberto Bolaño (en el 2004 todavía representado informalmente por Anagrama, en la actualidad por Andrew Wylie), Jorge Franco (Casanovas \& Lynch), Rodrigo Fresán (Casanovas \& Lynch), Santiago Gamboa (Schavelzon y Graham), Gonzalo Garcés (Schavelzon y Graham), Fernando Iwasaki (Silvia Bastos), Mario Mendoza (Schavelzon y Graham), Ignacio Padilla (Antonia Kerrigan), Edmundo Paz-Soldán (Schavelzon y Graham), Cristina Rivera Garza (desde 2015 Verónica Flores, México), Iván Thays (Schavelzon y Graham) y Jorge Volpi (Antonia Kerrigan).
} 
A estos indicios, que reproducen la matriz productiva del boom, habría que añadir el insistente énfasis en la novela como género privilegiado y excluyente. Para la literatura latinoamericana mundial, no importa cuánta poesía, cuánto ensayo o cuánta narrativa breve se produzca en los dominios locales, pues la única forma que va a ser considerada "literatura" es la novela en un formato más o menos clásico, más o menos europeo, más o menos decimonónico. Así, todos los participantes del Primer Encuentro de "Autores" Latinoamericanos son conocidos ante todo como novelistas, lo que da a entender que para ser considerado “autor", por lo pronto en el ritual llevado a cabo en Sevilla, era condición necesaria escribir y publicar novelas. Tampoco en este punto, que desde luego también reproduce ciertas pautas del boom, Cabrera Infante deja lugar para dudas: "para desmentir al crítico literario que enunció: 'América, novela sin novelistas.' Ahora era América con demasiados novelistas” (10).

Esta serie de atributos o señas de admisión, en la que con algunas excepciones coincide la mayoría de los participantes del ritual y que para las reglas de la literatura latinoamericana mundial favorece la identificación como "autor" (novelista, publicado en España en alguna editorial influyente y en el mejor de los casos reconocido con alguno de sus premios, residente fuera de América Latina y con agente acreditado en el dominio internacional), reduce el espectro de los potenciales invitados significativamente hasta quedar delimitado por los escritores, aspirantes a integrar o consolidarse en la literatura latinoamericana mundial, que ya han sido previamente seleccionados por la industria cultural de la metrópoli. Es, acaso, este recorte previo el que permite explicar las siguientes palabras del prólogo de Cabrera Infante: “Adolfo García Ortega ha convocado otros demonios al reunir a todos -o casi todos- los escritores jóvenes de América en un evento cuyo éxito literario es patente. Seix Barral reunió en Sevilla a todos o aparentemente todos los escritores jóvenes de América que escriben en español” (12). El sintagma “todos los escritores jóvenes de América”, si se ubica en el marco implícito que acabo de desglosar, sorprende menos de lo que lo hace a primera vista, pues para valer como "escritor joven de América”, en el marco internacional en el que tuvo lugar el evento, se debe cumplir con la serie de condiciones habilitantes que ya he expuesto. Solo visto de este modo no extraña que, para Cabrera Infante, “todos” los escritores jóvenes de América sean once y que, de esos once, solo una sea mujer.

$\mathrm{Al}$ otro lado, frente a los escritores que se sucedieron con sus exposiciones, se encontraban los periodistas culturales españoles. Cabrera Infante, retoma esta información en su prólogo y reproduce, en los siguientes términos, una nota al respecto aparecida en El Diario de Sevilla: “'Sevilla. El Encuentro de Autores Latinoamericanos, promovido por la editorial Seix-Barral y celebrado en la sede de la Fundación Lara, se clausuró ayer con un interesante careo' -careo era el 
nombre correcto para el evento- 'entre escritores del otro lado del charco y responsables de los suplementos especializados de información cultural”' (13). La participación de los periodistas, en principio, puede ser explicada por razones publicitarias: van a ser ellos los responsables de dar a conocer entre un público más amplio los debates llevados a cabo en el evento. Al mismo tiempo, sin embargo, habría que considerar el término "careo" con el que tanto la nota del periódico como Cabrera Infante caracterizan la interacción entre público y expositores. Los periodistas españoles, si se le asigna cierta densidad semántica al término "careo", parecen haber asistido al evento con una postura o representación relativa al objeto cultural en cuestión que debía ser desmitificada por los expositores; se trataba, se deduce, de rebatir sus preconceptos en relación con la literatura latinoamericana. Para eso, para corregir una imagen falsa o anquilosada de la literatura latinoamericana entre los periodistas españoles fue montado el encuentro. Una pregunta oportuna, y acaso natural, con la que se podría seguir es por qué la literatura latinoamericana le tiene que dar explicaciones al periodismo español. Desde ya que hay razones comerciales comprometidas y que los escritores invitados quisieron informar sobre un "giro" o "recambio" en la literatura de América Latina, pero ante todo habría que considerar que la construcción de un escenario y de un "careo" de este tipo responden, en realidad, a las dinámicas propias del dominio de la literatura latinoamericana mundial. Las explicaciones que fueron a ofrecer los escritores se corresponde no exactamente con los avatares de las literaturas locales sino con la mucho más necesitada de negociaciones y condicionada por demandas exógenas del nivel mundial. Los escritores, visto de este punto de vista, no fueron exactamente a informar sobre la situación de la literatura en América Latina sino sobre lo que el periodismo cultural y el público internacionales pueden esperar de la fracción que va a entrar -o que aspira a hacerlo- en circulación mundial.

Ahora bien, ¿cuál fue el núcleo temático del encuentro y sobre qué versaron las exposiciones? En la línea que ya habían inaugurado algunos años atrás El crack y McOndo, los participantes dieron cuenta de la evolución de la literatura latinoamericana reciente y, en general, reclamaron para sí la posibilidad de apartarse del paradigma del realismo mágico como estética oficial de América Latina. Este reclamo, compartido por todos los expositores, es el que le asignó al evento un carácter polémico. Frente a la representación anquilosada que supone que los escritores latinoamericanos apelan o deben apelar al color local como marca distintiva de su universo literario, los escritores reunidos en Sevilla argumentaron en favor de una escritura despegada de esa y de cualquier otra prescripción. Su distanciamiento de la norma marcada por consignas identitarias y el alegato en favor de una pertenencia a la literatura universal sin adjetivaciones nacionales o regionales se manifiesta, además, en la práctica escrituraria 
concreta que estos escritores ya habían comenzado a divulgar desde mediados de los años 90 y que insistía en situar sus relatos fuera de escenarios latinoamericanos “típicos" o pintorescos, ya sean estos lugares identificables como empíricamente fuera del subcontinente o susceptibles de ser caracterizados como lugares genéricos o no-lugares, según la terminología de Marc Augé. Fernando Iwasaki, por ejemplo, hizo referencia a este tipo de desplazamiento en los siguientes términos:

Los mexicanos Jorge Volpi e Ignacio Padilla tienen excelentes novelas ambientadas en Suiza, Francia y Alemania; el boliviano Edmundo Paz Soldán es autor de una obra que transcurre en el campus de Madison; el peruano Iván Thays construye en Busardo su propio territorio literario y mediterráneo; el colombiano Santiago Gamboa nos demuestra en Los impostores que "siempre nos quedará Pekín"; y el chileno Roberto Bolaño lo mismo ambienta sus novelas en París o el Distrito Federal mexicano, escenario de la fastuosa Mantra de Rodrigo Fresán, quien ahora mismo persigue a sus personajes por los jardines de Kensington. ¿Y qué decir de las ficciones japonesas de Mario Bellatín o de los paraísos magrebíes de Ruy Sánchez, por no hablar de los desterrados italianos del ecuatoriano Leonardo Valencia, de las intrigas saharianas del argentino Alfredo Taján, o del esperpento español del venezolano Juan Carlos Méndez Guédez? (120)

Pero quien pretendió ir al meollo de la cuestión es Jorge Volpi. La ubicación de un relato en un escenario "característico" o en uno no asociado con América Latina, al menos directamente, en realidad debería ser comprendida como una cuestión de acabado, pues en el fondo de lo que se trata es de una apuesta por la tradición cosmopolita, por oposición a una de corte nativista. Los escritores "jóvenes" de Sevilla vienen, así, a confrontar el imaginario que Volpi denomina "nacionalista" -aunque tal vez podríamos decir "regionalista"- para reclamar un lugar entre las filas del cosmopolitismo universal. Según Volpi articula su exposición, este posicionamiento debe ser defendido frente a la crítica académica latinoamericanista, encarnada en su exposición por un profesor ficticio de nombre Ignatius Berry. Su alegato del cosmopolitismo lo conduce a recordar afirmativamente que "los mejores escritores latinoamericanos han pertenecido siempre, casi sin excepciones, al grupo de los “cosmopolitas”" (“El fin...” 218) sin dejar de señalar que la contienda no es nueva sino que "en América Latina siempre han coexistido estos dos bandos irreconciliables: los 'nacionalistas' y los 'cosmopolitas"” (216).

Ahora bien, un pequeño ejercicio de reubicación de las exposiciones en la situación localizada en la que efectivamente tuvieron lugar -en Sevilla, en la fundación Lara, frente a periodistas españoles- permite comprender más acabadamente quién es en realidad el interlocutor ideal y cuál era la finalidad más inmediata del ritual. Los escritores de Sevilla estaban en el encuentro para tomar el relevo en la literatura latinoamericana mundial, esto es, para, bajo tutelaje de 
Seix Barral/Planeta, inscribirse en la estela del boom bajo consignas actualizadas. El interlocutor al que buscaban interpelar, representado por los periodistas, era el consumidor internacional, por lo pronto español, habituado a pautas estéticas acuñadas durante el apogeo de García Márquez y Vargas Llosa durante los años 60 y al que ahora se le estaba proponiendo una renovación del pacto. La "polémica", lejos de estar dirigida a desestructurar a alguna crítica latinoamericanista, debe ser entendida como un acto público con el que se busca llamar la atención y producir condiciones favorables para una reconfiguración del horizonte de expectativas de la literatura latinoamericana mundial que, con la oferta de los epígonos del boom de los años 80 -la de Isabel Allende, Ángeles Mastretta, Laura Esquivel, etc.-, había ingresado en un bucle de agotamiento. En otras palabras, la apuesta por el cosmopolitismo o por la liberación de constricciones regionalistas en realidad debe ser considerada como un recurso para superar tal empantanamiento y liberar vetas de mercado alternativas.

Esta "polémica", vale aclarar, no responde a la evolución o a las necesidades de los escenarios latinoamericanos locales. El debate cosmopolitismo/regionalismo, como recuerda Volpi, no es nada novedoso y, por eso mismo, poco tiene que ver con la actualidad de las literaturas locales de América Latina, aunque sí -como veremos-mucho con la proyección de América Latina hacia el mundo o más estrictamente con la literatura latinoamericana mundial.

La tradición cosmopolita en América Latina -si se la quiere caracterizar de tal modo- ha dicho lo suyo y nadie duda de su aporte tanto al corpus más específico como a la literatura universal. Por esta razón, no necesita que hoy en día alguien abogue por ella. Como ha recordado recientemente Christopher Domínguez en abierta polémica con los escritores de El crack, la escritura desprovista de color local se encuentra presente en toda la historia de la literatura latinoamericana, pero nunca ha requerido presentar alegatos sensacionalistas o desatar polémicas: "Me hubiera bastado con decir que Farabeuf, o la crónica de un instante (1965), de Salvador Elizondo, nada tiene que ver con México pero el autor de una de nuestras grandes novelas, un verdadero moderno, no se preocupaba por esas tarugadas". Aunque cierta zona importante de la demanda internacional tal vez no le haya dado una buena acogida, o no tan buena como al regionalismo magicorrealista de García Márquez y luego al de Isabel Allende, el cosmopolitismo latinoamericano no es de ningún modo una invención reciente y tampoco está en el centro de los debates en las tramas locales del subcontinente.

Para comprender mejor el ritual de Sevilla, por lo tanto, conviene ubicarlo en el circuito de producción de literatura latinoamericana mundial donde el paradigma del realismo mágico, ya entrado el siglo XXI, parecía agotado y condenado a una lenta agonía mediante repetición. El grupo Planeta, en colaboración con otros actores calificados del escenario internacional, asume, entonces, como 
causa propia una renovación del mercado y una actualización de las pautas de recepción. Los escritores reclutados, en tanto "jóvenes" aspirantes a encarnar el relevo, por su parte, van a escenificar el intento de reconfiguración del mercado en términos de "ruptura" con la tradición "regionalista" y en favor de los postulados del "cosmopolitismo".

Al margen de que, como señala Sarah Brouillette, ${ }^{2}$ este "cosmopolitismo" está en sintonía con el proceso contemporáneo de reconfiguración geopolítica global, lo que me interesa señalar es que la "polémica” reproduce patrones que ya estaban presentes en las clásicas discusiones entre Julio Cortázar y José María Arguedas, David Viñas y Julio Cortázar o Mario Vargas Llosa y José María Arguedas (cfr., por ejemplo, Moraña Arguedas/Vargas Llosa) y tiene que ver con las tensiones naturales que se generan durante el proceso de inscripción de las literaturas periféricas en las corrientes de la literatura mundial, porque, en realidad, lo que sucede, como bien ha argumentado Antonio Candido, es que tanto una codificación como la otra solo pueden surgir de realidades colonizadas económica, política y culturalmente. Tanto el "cosmopolitismo" como el "regionalismo" toman como punto de referencia la realidad dominante a la que no dejan de apuntar como consumidor y evaluador privilegiado. El horizonte de expectativas de la metrópoli, puesto que los mercados locales presentan carencias estructurales severas, es el marco de referencia al que responden las literaturas colonizadas. Esta dependencia de la metrópoli, de acuerdo con Candido, debe ser entendida como "retraso":

Retraso que estimula la copia servil de todo cuanto la moda de los países adelantados ofrece, además de seducir a los escritores con la migración, exterior e interior. Retraso que propone lo que hay de más peculiar en la realidad local, insinuando un regionalismo que, al parecer afirmación de la identidad nacional, puede ser en verdad un modo insospechado de ofrecer a la sensibilidad europea el exotismo que ella deseaba, como distracción; y que así se vuelve forma más aguda de dependencia que la independencia. Desde la perspectiva actual, parece que las dos tendencias son solidarias y nacen de la misma situación de retraso o subdesarrollo. (349)

De aquí se concluye, si se le da crédito a Candido, que el ritual llevado a cabo en Sevilla, y en consideración de su localización geográfica y del actor proveedor de infraestructura y recursos económicos, donde los escritores enarbolaron la bandera del cosmopolitismo, pero no la de una escritura soberana, liberada en ningún aspecto del punto de referencia eurocéntrico, fue, en última instancia, también -o por lo pronto- un acto de confirmación de las viejas ataduras coloniales.

2 "In effect, in a double movement, contemporary writers are successfully marketed through their inscription in a lengthy cosmopolitan tradition, and, meanwhile, this constructed tradition, in supporting the image of an inevitably networked globalized world, is inseparable from the power of global capital” (94). 